\title{
On Cover-Avoiding Subgroups of Sylow Subgroups of Finite Groups
}

\author{
YANGMING LI (*) \\ Dedicated to Professor Shirong Li on the occasion \\ of his seventieth birthday.
}

ABSTRACT - A subgroup $H$ of a finite group $G$ is called to have the cover-avoidance property in $G, H$ is a CAP subgroup of $G$ in short, if $H$ either covers or avoids every chief factor of $G$. In the present work, we fix a subgroup $D$ in every Sylow subgroup $P$ of $F^{*}(G)$ satisfying $1<|D|<|P|$ and study the structure of $G$ under the assumption that every subgroup $H$ with $|H|=|D|$ has the cover-avoidance property in $G$. We state our results in the broader context of formation theory.

\section{Introduction.}

All groups considered in this paper are finite. We use conventional notions and notation, as in Huppert[11]. $G$ always denotes a finite group, $|G|$ the order of $G, \pi(G)$ the set of all primes dividing $|G|, G_{p}$ a Sylow $p$-subgroup of $G$ for some $p \in \pi(G)$.

A class $\mathcal{F}$ of finite groups is called a formation if $G \in \mathcal{F}$ and $N \unlhd G$ then $G / N \in \mathcal{F}$, and if $G / N_{i}(i=1,2) \in \mathcal{F}$ then $G / N_{1} \cap N_{2} \in \mathcal{F}$. If, in addition, $G / \Phi(G) \in \mathcal{F}$ implies $G \in \mathcal{F}$, we say that $\mathcal{F}$ to be saturated. An interesting example of saturated formation is the class of all supersolvable groups, which is denoted by $\mathcal{U} . Z_{\infty}^{\mathcal{U}}(G)$ denotes the $\mathcal{U}$-hypercenter of $G$, that is, the product of all normal subgroups of $G$ whose $G$-chief factors are of prime order.

DeFinition 1.1. Let $L / K$ be a chief factor of $G$ and $H$ a subgroup of $G$. We say that

(*) Indirizzo dell'A.: Dept. of Math., Guangdong Institute of Education, Guangzhou, 510310, China.

E-mail: liyangming@gdei.edu.cn

Project supported in part by NSFC (10871210) and NSF of Guangdong (06023728).

2000 Mathematics Subject Classification: 20D10, 20D20. 
i) $H$ covers $L / K$ if $L \leq H K$;

ii) $H$ avoids $L / K$ if $L \cap H \leq K$;

iii) $H$ has the cover-avoidance property in $G, H$ is a CAP subgroup of $G$ in short, if $H$ either covers or avoids every chief factor of $G$.

The cover-avoidance property of subgroup was first studied by Gaschütz in [7] to study the solvable groups, later by Gillam [8] and Tomkinson [18]. Clearly normal subgroups are CAP subgroups. Examples of CAP subgroups in the universe of solvable groups are wellknown. The most remarkable CAP subgroups of a solvable group are perhaps the Hall subgroups [7]. By a obvious consequence of the definition of supersolvable group every subgroup of supersolvable group is a CAP subgroup. Ezquerro has given the following converse argument (see [[5], Theorem D]): if there exists a normal solvable subgroup $E$ of $G$ such that $G / E$ is supersolvable and all maximal subgroups of any Sylow subgroups of $F(E)$ are CAP subgroups of $G$, then $G$ is supersolvable. In [1], Asaad said it is possible to extend Ezquerro's result with formation theory. In [14], the authors replaced the Fitting subgroup by the generalized Fitting subgroup so that the hypothesis of the solvability was removed in the result above, that is, Let $\mathcal{F}$ be a saturated formation containing $\mathcal{U}$. Suppose that $E$ is a normal subgroup of $G$ such that $G / E \in \mathcal{F}$. Suppose that all maximal subgroups of the Sylow subgroups of $F^{*}(E)$, where $F^{*}(E)$ is the generalized Fitting subgroup of $E$, are CAP subgroups of $G$. Then $G \in \mathcal{F}$ ([14], Corollary 4.3). In [21], the authors have given many characterizations of solvable group or $p$ solvable group or supersolvable group $G$ under assumption that some certain subgroups of $G$ are CAP subgroups of $G$. In [2], A subgroup $H$ of $G$ is said to be a strong CAP-subgroup of $G$ if $H$ is a CAP-subgroup of any subgroup of $G$ containing $H$. The main result of [2] is as follows: Let $\mathcal{F}$ be a saturated formation containing $\mathcal{U}$ and $E$ a normal subgroup of $G$ such that $G / E \in \mathcal{F}$. Suppose that, for every non-cyclic Sylow subgroup $P$ of $F^{*}(E), P$ has a subgroup $D$ such that $1<|D|<|P|$ and all subgroups $H$ of $P$ with order $|H|=|D|$ and with order $|H|=2|D|$ (if $P$ is a non-abelian 2-group) are strong CAP-subgroups of $G$. Then $G \in \mathcal{F}$. In this paper, we go further in this direction to give a new result which covers the results mentioned above.

Main Theorem. Let $\mathcal{F}$ be a saturated formation containing $\mathcal{U}$ and $E$ a normal subgroup of $G$ such that $G / E \in \mathcal{F}$. Suppose that, for every non-cyclic Sylow subgroup $P$ of $F^{*}(E), P$ has a subgroup $D$ such that 
$1<|D|<|P|$ and all subgroups $H$ of $P$ with order $|H|=|D|$ and with order $|H|=2|D|$ (if $P$ is a non-abelian 2-group and $|P: D|>2$ ) satisfy the cover-avoidance property in $G$. Then $G \in \mathcal{F}$.

REMARK 1. Our result, together with the main result in [2], was stimulated by the Skiba's results in the nice paper [17], which is analogous to our main result by replacing the cover-avoidance property by weakly spermutability. There are some generalizations of Skiba's results, such as that in [13], [15]. However it is easy to find solvable groups with CAP subgroups which are not weakly s-permutable subgroup. Conversely, there are also groups with weakly s-permutable subgroup which are not CAP subgroups (ref. [[5], Example 5]).

Recently, Fan, Guo and Shum introduced the semi-cover-avoidance property [6], which is the generalization not only of the cover-avoidance property but also of c-normality [19].

DEFINITION 1.2 ([6]). A subgroup $H$ of $G$ is said to have the semi-coveravoidance property in $G$ if there exists a chief series of $G$,

$$
1=G_{0} \leq G_{1} \leq \cdots \leq G_{n}=G
$$

such that $H$ either covers or avoids chief factor $G_{i+1} / G_{i}$ for any $i \in\{0,1, \cdots, n-1\}$. In this case $H$ is called a SCAP subgroup of $G$.

REMARK 2. In [3], SCAP subgroup was named partial CAP-subgroup.

For the sake of convenience of statement, we introduce the following notation.

Let $P$ be a $p$-subgroup of $G$ for some prime $p$. We say that $P$ satisfies $\diamond_{1}$ $\left(\diamond_{2}\right.$ respectively) in $G$ if

$\left(\diamond_{1}\right)$ : $P$ has a subgroup $D$ such that $1<|D|<|P|$ and all subgroups $H$ of $P$ with order $|H|=|D|=d_{p}$ and with order $|H|=2|D|=2 d_{p}$ (if $P$ is a non-abelian 2-group and $|P: D|>2$ ) satisfy the cover-avoidance property in $G$.

$\left(\diamond_{2}\right)$ : $P$ has a subgroup $D$ such that $1<|D|<|P|$ and all subgroups $H$ of $P$ with order $|H|=|D|=d_{p}$ and with order $|H|=2|D|=2 d_{p}$ (if $P$ is a non-abelian 2-group and $|P: D|>2$ ) satisfy the semi-cover-avoidance property in $G$. 


\section{Preliminaries.}

Lemma 2.1 ([16]). Let $S$ be a CAP subgroup of $G$ and $N$ a normal subgroup of $G$. Then

(1) $N$ is a CAP subgroup of $G$;

(2) $S N / N$ is a CAP subgroup of $G / N$;

(3) $S N$ is a CAP subgroup of $G$;

(4) $S \cap N$ is a CAP subgroup of $G$.

Lemma 2.2 ([6], [10]). Let $S$ be a SCAP subgroup of $G$. Then

(1) Every CAP subgroup of $G$ is a SCAP subgroup of $G$;

(2) If $S \leq K \leq G$, then $S$ is a $S C A P$ subgroup of $K$;

(3) If $N \leq S$ and $N \unlhd G$, then $S / N$ is a $S C A P$ subgroup of $G / N$;

(4) If $N \unlhd G$ and $(|S|,|N|)=1$, then $S N / N$ is a $S C A P$ subgroup of $G / N$.

LEMMA 2.3. Every minimal normal subgroup of $G$ is a minimal $C A P$ subgroup of $G$.

Proof. Suppose that $N_{1}$ is a proper subgroup of $N$ which is a CAP subgroup of $G$. Then $N_{1}$ either covers or avoids $G$-chief factor $N / 1$. If $N_{1}$ covers $N / 1$, then $N_{1}=N$, a contradiction. If $N_{1}$ avoids $N / 1$, then $N_{1}=N_{1} \cap N=1$. Therefore $N$ is a minimal CAP subgroup of $G$.

Lemma 2.4. Let $P$ be a normal $p$-subgroup of $G$ and $\Phi(P)=1$ for some prime $p$. If $P$ satisfies $\diamond_{1}$ in $G$, then every $G$-chief factor of $P$ is a cyclic group of prime order, in another word, $P \leq Z_{\infty}^{\mathcal{U}}(G)$.

Proof. Suppose that $N$ is a minimal normal subgroup of $G$ contained in $P$. Let $U$ be a complement of $N$ in $P$. It follows from Lemma 2.3 that $|N| \leq|D|=d_{p}$. So we can pick a maximal subgroup $M$ of $N$ and a subgroup $L$ of $U$ such that $|L M|=d_{p}$. Hence $L M$ is a CAP subgroup of $G$ by the hypotheses. Thus $M=L M \cap N$ is a CAP subgroup of $G$ by Lemma 2.1 (4). Lemma 2.3 implies that $M=1$. Therefore $|N|=p$. So $N \leq Z_{\infty}^{\mathcal{U}}(G)$.

Consider the factor group $G / N$. If $d_{p}>p$, then $1<D / N<P / N$. It follows from Lemma 2.1 (2) that $P / N$ satisfies $\diamond_{1}$ in $G / N$. Hence $G / N$ satisfies the hypotheses of the lemma. Therefore $P / N \leq Z_{\infty}^{\mathcal{U}}(G / N)=$ $=Z_{\infty}^{\mathcal{U}}(G) / N$ by induction. Hence $P \leq Z_{\infty}^{\mathcal{U}}(G)$, as desired. Now assume that $d_{p}=p$. Take an arbitrary subgroup $T / N$ of $P / N$ of order $p$. Since $\Phi(P)=1, T=T_{1} N$ where $T_{1}$ is a subgroup of $T$ with prime order. Hence 
$T_{1}$ is a CAP subgroup of $G$ by the hypotheses. Thus $T_{1} N / N=T / N$ is a CAP subgroup of $G / N$ by Lemma 2.1 (2). So $P / N$ satisfies $\nabla_{1}$ in $G / N$. Hence $P \leq Z_{\infty}^{\mathcal{U}}(G)$ by induction.

The generalized Fitting subgroup $F^{*}(G)$ of $G$ is the unique maximal normal quasinilpotent subgroup of $G$. Its definition and important properties can be found in ([12], X, 13). We would like to give the following basic facts we will use in our proof.

Lemma 2.5. Let $G$ be a group and $N$ a subgroup of $G$.

(1) If $N$ is normal in $G$, then $F^{*}(N) \leq F^{*}(G)$;

(2) $F^{*}(G) \neq 1$ if $G \neq 1$; in fact, $F^{*}(G) / F(G)=\operatorname{Soc}\left(F(G) C_{G}(F(G)) / F(G)\right)$;

(3) $F^{*}\left(F^{*}(G)\right)=F^{*}(G) \geq F(G)$; if $F^{*}(G)$ is solvable, then $F^{*}(G)=F(G)$;

(4) $C_{G}\left(F^{*}(G)\right) \leq F(G)$;

(5) Let $N=Z(E(G)) \Phi(F(G))$. Then $F^{*}(G / N)=F^{*}(G) / N$, where $E(G)$ is the layer of $G$;

(6) Suppose that $P$ is a normal subgroup of $G$ contained in $O_{p}(G)$. Then $F^{*}(G / \Phi(P))=F^{*}(G) / \Phi(P)$.

Proof. (1)-(4) please see ([12], X, 13); (5) is ([9], Proposition 4.10). (6) is a corollary of (5).

Lemma 2.6. Let $p$ be the smallest prime dividing the order of $F^{*}(G)$ and $P$ a Sylow p-subgroup of $F^{*}(G)$. Suppose that $P$ satisfies $\diamond_{2}$ in $G$. Then $F^{*}(G)$ is solvable.

Proof. Assume that the lemma is false and choose $G$ to be a counterexample of the smallest order. By the well-known Odd Order FeitThompson Theorem, we have $p=2$.

Suppose that $F^{*}(G)<G$. Since $P$ satisfies $\diamond_{2}$ in $G, P$ satisfies $\diamond_{2}$ in $F^{*}(G)$ by Lemma 2.2 (2). The minimal choice of $G$ implies that $F^{*}\left(F^{*}(G)\right)=F^{*}(G)$ is solvable, a contradiction. Therefore $F^{*}(G)=G$. This is to say that $G$ is a quasinilpotent group. If $F(G) \neq 1$, then take a minimal normal subgroup $N$ of $G$, such that $N \leq F(G)$. Then $N$ is an elementary abelian $q$-group for some prime $q$. Since the class of quasinilpotent groups is a formation (see Remark IX 2.7 in [4]), then factor group $G / N$ is also quasinilpotent. This is to say that $F^{*}(G / N)=G / N=F^{*}(G) / N$. Now if $q$ is odd, then $P N / N$ satisfies $\diamond_{2}$ in $G / N$ by Lemma $2.2(4)$ and then $G / N$ is solvable by the minimal choice of $G$. This implies that $G$ is solvable, a 
contradiction. Hence $q=2$. If $d_{2}>2$, then $P / N$ satisfies $\diamond_{2}$ in $G / N$ by Lemma 2.2(3). The minimal choice of $G$ implies that $G / N$ is solvable and so is $G$, again a contradiction. Hence $d_{2}=2$. In this case $G$ is 2 -nilpotent by Theorem 3.8 of [10]. Since 2-nilpotent groups are solvable by the OddOrder Feit-Thompson Theorem, we have again a contradiction. Therefore $F(G)=1$. By Theorem X.13.18 of [12], the group $G$ is a direct product of non-abelian simple group and therefore every chief factor of $G$ is a nonabelian simple group. No 2-subgroup can cover a non-abelian chief factor of $G$. Thus, if $H$ is a subgroup of $P$ of order $d_{2}$, then $H$ avoids every chief factor of some chief series of $G$. This is to say that $d_{2}=1$. This is the final contradiction.

\section{The Proof of Main Theorem.}

Assume that the theorem is false and choose $G$ to be a counterexample of the smallest order.

(1) $F^{*}(E)$ is solvable and $F^{*}(E)=F(E)$.

Let $p$ be the smallest prime dividing the order of $F^{*}(E)$ and $P$ a Sylow $p$ subgroup of $F^{*}(E)$. If $P$ is cyclic, then $F^{*}(G)$ is $p$-nilpotent by ([11], IV, Satz 2.8). So $F^{*}(E)$ is solvable and $F^{*}(E)=F(E)$. Hence assume that $P$ is noncyclic. By the hypotheses, $P$ satisfies $\diamond_{1}$ in $G$. Hence $P$ satisfies $\diamond_{2}$ in $G$ by Lemma 2.2(1). Applying Lemma 2.6, we have $F^{*}(E)$ is solvable and $F^{*}(E)=F(E)$.

(2) There exists a prime $q \in \pi\left(F^{*}(E)\right)$ such that some $G$-chief factors $L / K$ of $O_{q}(E)$ are not of prime order. Furthermore, $O_{q}(E)$ is not cyclic and $\Phi\left(O_{q}(E)\right) \neq 1$.

If otherwise, then we have $F^{*}(E)=F(E) \leq Z_{\infty}^{\mathcal{U}}(G)$. Then $G / C_{G}\left(F^{*}(E)\right) \in$ $\in \mathcal{F}$ by Theorem 6.10 of Chapter IV in [4]. It follows that $G / C_{E}\left(F^{*}(E)\right)=$ $=G / E \cap C_{G}\left(F^{*}(E)\right) \in \mathcal{F}$. Thus $G / F(E) \in \mathcal{F}$ by Lemma 2.5 (4). Since every $G$ chief factor of $F(E)$ is of prime order and $\mathcal{F}$ contains the class of all supersolvable groups, $G \in \mathcal{F}$ which is a contradiction.

By Lemma 2.4, we have $\Phi\left(O_{q}(E)\right) \neq 1$.

(3) Suppose that $\Phi\left(O_{q}(E)\right) \neq 1$ for some $q \in \pi\left(F^{*}(E)\right)$. Take a minimal normal subgroup $N$ of $G$ contained in $\Phi\left(O_{q}(E)\right)$. Then $|N|=|D|=d_{q}$.

By Lemma 2.3 we have $|N| \leq|D|$. If $|D|>|N|$, then consider the factor group $G / N$. Then $F^{*}(E / N)=F^{*}(E) / N$ by Lemma 2.5 (6). Now Lemma 2.1 
(2) implies that $(G / N, E / N)$ satisfy the hypotheses of the theorem. It follows that $G / N \in \mathcal{F}$ by the minimal choice of $G$. So $G \in \mathcal{F}$, a contradiction. Therefore $|N|=|D|=d_{q}$.

(4) If $\Phi\left(O_{q}(E)\right) \neq 1$, then $|D|=d_{q}=q$.

Take a minimal normal subgroup $N$ of $G$ contained in $\Phi\left(O_{q}(E)\right)$. Then $|N|=|D|=d_{q}$ by (3). Now we claim that $|N|=d_{q}=q$.

Pick a maximal subgroup $M$ of $N$ such that $M \unlhd O_{q}(E)$. If there is another minimal subgroup $T / M$ of $O_{q}(E) / M$ different to $N / M$. Then $|T|=|N|=d_{q}$. Hence $T$ is a CAP subgroup of $G$ by hypotheses. Thus $M=T \cap N$ is also a CAP subgroup of $G$ by Lemma 2.1 (4). Hence $|N|=q$ by Lemma 2.3, as desired. So we assume that $N / M$ is the unique minimal subgroup of $O_{q}(E) / M$. Take a minimal normal subgroup $L / N$ of $O_{q}(E) / N$. Then $|L / M|=q^{2}$ and $N / M$ is also the unique minimal subgroup of $L / M$. Therefore $L / M$ is a cyclic group of order $q^{2}$. Denote $L / M=\langle a M\rangle$. Since $a^{q} \in \Phi(L) \leq N$ and $\exp (N)=q$, we have $L=M\langle a\rangle$ and $o(a)=q^{2}$. If $\Phi(L)=N$, then $L=M\langle a\rangle=\langle a\rangle$. Hence $N=\left\langle a^{q}\right\rangle$ is of prime order. So we assume that $\Phi(L)<N$. Since $L$ is normal in $O_{q}(E), \Phi(L)$ is normal in $O_{q}(E)$. Hence we can pick a maximal subgroup $M_{1}$ of $N$ containing $\Phi(L)$ such that $M_{1} \unlhd O_{q}(E)$. Since $a^{q} \in \Phi(L) \leq M_{1}$, we have $\left|M_{1}\langle a\rangle\right|=|N|=d_{q}$. By the hypotheses, $M_{1}\langle a\rangle$ is a CAP subgroup of $G$. Then $M_{1}=N \cap M_{1}\langle a\rangle$ is a CAP subgroup of $G$ by Lemma 2.1 (4). It follows from Lemma 2.3 that $|N|=q$.

(5) Final contradiction.

By (2) we know that there exists a prime $q \in \pi\left(F^{*}(E)\right)$ such that some $G$ chief factors $L / K$ of $O_{q}(E)$ are not of prime order. We may choose $L / K$ satisfying that every $G$-chief factor $T / W$ is of prime order with $T<L$.

If there is some element $x \in L \backslash K$ with $o(x)=q$ or $o(x)=4$, then $\langle x\rangle$ is a CAP subgroup of $G$ by the hypotheses of the theorem and (4). It follows from Lemma 2.1 (2) that $\langle x\rangle K / K$ is a CAP subgroup of $G / K$. By Lemma 2.3, we have $L / K=\langle x\rangle K / K$ which contradicts with the choice of $L / K$. Therefore all cyclic subgroups of order $q$ or order 4 of $L$ are contained in $K$. Suppose that we have a $G$-chief series of $O_{q}(E)$ as follows

$$
1=U_{0} \unlhd U_{1} \unlhd \cdots \unlhd U_{n}=K \unlhd L .
$$

Then $\left|U_{i} / U_{i-1}\right|=q$ for $i \in\{1,2, \cdots, n\}$ by the choice of $L / K$. Thus $G / C_{G}\left(U_{i} / U_{i-1}\right)$ is an Abelian group of exponent dividing $q-1$. Denote

$$
X=\bigcap_{i=1}^{n} C_{G}\left(U_{i} / U_{i-1}\right) .
$$


Then $G / X$ is an Abelian group of exponent dividing $q-1$. Let $R$ be a $q^{\prime}$ subgroup of $X$. Then $R$ centralizes $K$ and so $R$ centralizes all elements of $L$ of order $q$ and order 4 . Then $R$ centralizes $L$ by Satz IV.5.12 of [11]. Thus $X / C_{X}(L / K)$ is a $q$-group. Then $X C_{G}(L / K) / C_{G}(L / K)$ is a normal $q$ subgroup of $G / C_{G}(L / K)$. Hence $X C_{G}(L / K) / C_{G}(L / K)=1$ by Corollary 6.4 of [20] (page 221). So $X \leq C_{G}(L / K)$. This implies that $G / X$ acts irreducibly on $L / K$. So $|L / K|=q$ by Lemma 1.3 of [20] (page 3), the final contradiction.

The proof of the theorem is now complete.

\section{Final Remarks.}

Many known results are special cases of our Main Theorem and one also can obtain several new results from our Main Theorem.

CoROLlaRY 4.1. Let $\mathcal{F}$ be a saturated formation containing $\mathcal{U}$ and $E$ a normal subgroup of $G$ such that $G / E \in \mathcal{F}$. Suppose for any every noncyclic Sylow subgroup $P$ of $F^{*}(E)$ at least one of the following holds:

(1) every maximal subgroup of $P$ is a CAP subgroup of $G$;

(2) every cyclic subgroup of $P$ of prime order or order 4 (if $P$ is a 2group) is a CAP subgroup of $G$.

Then $G \in \mathcal{F}$.

COROLLARY 4.2 ([14]). Let $\mathcal{F}$ be a saturated formation containing $\mathcal{U}$ and $E$ a normal subgroup of $G$ such that $G / E \in \mathcal{F}$. Suppose for any every non-cyclic Sylow subgroup $P$ of $F^{*}(E)$, every maximal subgroup of $P$ is a $C A P$ subgroup of $G$. Then $G \in \mathcal{F}$.

CoRollary 4.3. Let $\mathcal{F}$ be a saturated formation containing $\mathcal{U}$ and $E$ a normal subgroup of $G$ such that $G / E \in \mathcal{F}$. Suppose for any every non-cyclic Sylow subgroup $P$ of $F^{*}(E)$, every cyclic subgroup of $P$ of prime order or order 4 (if $P$ is a 2-group) is a CAP subgroup of $G$. Then $G \in \mathcal{F}$.

CoROLLARY 4.4 ([1]). Let $\mathcal{F}$ be a saturated formation containing $\mathcal{U}$ and $E$ a solvable normal subgroup of $G$ such that $G / E \in \mathcal{F}$. Suppose that all maximal subgroups of non-cyclic Sylow subgroups of $F(E)$ are CAP subgroups of $G$. Then $G \in \mathcal{F}$. 
COROLLARY 4.5. Let $\mathcal{F}$ be a saturated formation containing $\mathcal{U}$ and $E$ a solvable normal subgroup of $G$ such that $G / E \in \mathcal{F}$. Suppose that all minimal subgroups and cyclic subgroup of order 4 of non-cyclic Sylow subgroups of $F(E)$ are CAP subgroups of $G$. Then $G \in \mathcal{F}$.

COROLLARY 4.6 ([5]). Let $E$ be a normal subgroup of $G$ such that $G / E$ is supersolvable. Then $G$ is supersolvable if $E$ is solvable and all maximal subgroups of the Sylow subgroups of $F(E)$ are CAP subgroups of $G$.

CoRollary 4.7 ([2], Theorem A). Let $\mathcal{F}$ be a saturated formation containing $\mathcal{U}$ and $E$ a normal subgroup of $G$ such that $G / E \in \mathcal{F}$. Suppose that, for every non-cyclic Sylow subgroup $P$ of $F^{*}(E)$, $P$ has a subgroup $D$ such that $1<|D|<|P|$ and all subgroups $H$ of $P$ with order $|H|=|D|$ and with order $|H|=2|D|$ ( if $P$ is a non-abelian 2-group) are strong CAPsubgroups of $G$. Then $G \in \mathcal{F}$.

Naturally we have following question:

QUESTION. Let $\mathcal{F}$ be a saturated formation containing $\mathcal{U}$ and $E$ a normal subgroup of $G$ such that $G / E \in \mathcal{F}$. Suppose that every non-cyclic Sylow subgroup $P$ of $F^{*}(E)$ satisfies $\diamond_{2}$ in $G$. Is then $G$ in $\mathcal{F}$ ?

Acknowledgments. The author is grateful to the referee for careful reading of the paper and for his or her useful suggestions which help him to improve the exposition.

\section{REFERENCES}

[1] M. AsaAD, On maximal subgroups of finite group, Comm. Algebra, 26 (11) (1998), pp. 3647-3652.

[2] A. Ballester-Bolinches - L. M. Ezquerro - A. N. Skiba, Subgroups of finite group with strong Cover-avoidance property, Bull. Austral. Math. Soc., 79 (2009), pp. 409-506.

[3] A. Ballester-Bolinches - L. M. Ezquerro - A. N. Skiba, Local embeddings of some families of subgroups of finite group, Acta Math. Sinica, Eng. Ser., 25 (2009), pp. 869-882.

[4] R. Doerk - T. Hawkes, Finite soluble groups. Berlin-New York: Walter De Gruyter, 1992.

[5] L. M. Ezquerro, A contribution to the theory of finite supersolvable groups, Rend. Sem. Mat. Univ. Padova, 89 (1993), pp. 161-170.

[6] Y. FAN - X. Y. GUO - K. P. SHUM, Remarks on two generalizations of 
normality of subgroups, Chinese Ann. Math., 27A (2) (2006), pp. 169-176 (in Chinese).

[7] W. Gaschütz, Praefrattinigruppen, Arch. Math., 13 (1962), pp. 418-426.

[8] J. D. GILlam, Cover-avoid subgroup in finite solvable groups, J. Algebra, 29 (1974), pp. 324-329.

[9] D. Gorenstein - R. Lyons - R. Solomon, The Classification of the Simple Finite Groups, Amer. Math. Soc., Providence Rhode Island, 1994.

[10] X. Y. Guo - P. F. Guo - K. P. Shum, On semi-cover-avoiding subgroups of finite groups, J. Pure Appl. Algebra, 209 (2007), pp. 151-158.

[11] B. HuPPERT, Endliche Gruppen I, Berlin-New York: Springer-Verlag, 1967.

[12] B. Huppert - N. BlackBurn, Finite Groups III, Berlin-New York: SpringerVerlag, 1982.

[13] Y. LI - S. QIAO - Y. WANG, On weakly s-permutably embedded subgroups of finite groups, Comm. Algebra, 37 (3) (2009), pp. 1086-1097.

[14] Y. Li - G. WANG, A note on maximal CAP subgroups of finite groups, Algebras, Groups and Geometries, 23 (2006), pp. 285-290.

[15] B. Li - Z. ZHANG, The influence of s-conditionally permutable subgroups on finite groups, Sci. China Ser. A, 52 (2) (2009), pp. 301-310.

[16] J. A. Petrillo, The cover-avoidance property in finite groups, $\mathrm{PhD}$ thesis, State University of New York at Binghamton, 2003.

[17] A. N. SkIBA, On weakly s-permutable subgroups of finite groups, J. Algebra, 315 (2007), pp. 192-209.

[18] M. J. Tomkinson, Cover-avoidance properties in finite solvable groups, Canad. Math. Bull., 19 (2) (1976), pp. 213-216.

[19] Y. WANG, C-normality of groups and its properties, J. Algebra, 180 (1996), pp. 954-965.

[20] M. Weinstein (editor), Between nilpotent and solvable, Polygonal Publishing House, Passaic (1982).

[21] Guo XIUYun - K. P. Shum, Cover-avoidance properties and the structure of finite groups, J. Pure Applied Algebra, 181 (2003), pp. 297-308.

Manoscritto pervenuto in redazione il 6 luglio 2009. 